\title{
EFEITOS DO MANEJO DE Eichhornia crassipes SOBRE A QUALIDADE DA ÁGuA EM Condições de Mesocosmos ${ }^{1}$
}

\author{
Effects of Waterhyacynth Management on Water Quality under Mesocosmic Conditions
}

\author{
MARTINS A.T. ${ }^{2}$ e PITELLI, R.A. ${ }^{3}$
}

\begin{abstract}
RESUMO - As macrófitas, apesar da enorme importância na dinâmica do ambiente aquático, quando formam extensas e densas colonizações, promovem uma série de prejuízos ao ambiente e aos usos múltiplos dos reservatórios. Nessas situações, há necessidade de redução de seu tamanho populacional, seja reduzindo as condições favoráveis ao crescimento, seja por meio do controle direto das plantas. Dentre as macrófitas aquáticas que promovem esses tipos de problema, o aguapé (Eichhornia crassipes) é considerada a mais importante. Seu controle é praticado em todo o mundo. O diquat tem sido bastante utilizado para o controle desta planta, em razão de seu baixo custo, eficácia, rapidez de controle e baixa toxicidade no ambiente aquático. O objetivo do presente trabalho foi avaliar os possiveis impactos causados pelo controle de Eichhornia crassipes sobre algumas características de qualidade da água em mesocosmos. Para isso, cinco situações experimentais foram estudadas: $\mathrm{CPCH}$ - mesocosmo colonizado por aguapé, o qual foi controlado pela aplicação do herbicida diquat; CPCG - mesocosmo colonizado por aguapé, o qual foi morto por congelamento; CPSH - mesocosmo colonizado com aguapé, sem controle; SPCH - mesocosmo sem macrófitas e com aplicação de diquat na superficie da água; e SPSH - mesocosmo sem macrófitas aquáticas e sem aplicação. O herbicida diquat foi utilizado na dose de 7,0 L da formulação comercial Reward/ha. A temperatura foi mais elevada nos mesocosmos sem plantas, devido à maior incidência de raios solares na coluna d'água. As concentrações de oxigênio dissolvido foram menores nos mesocosmos colonizados pelo aguapé e também tiveram rápida queda após o controle das plantas tanto com diquat como por congelamento. $\mathrm{O} \mathrm{pH}$ da água foi maior nos mesocosmos sem a cobertura da macrófita. Os valores de sólidos totais dissolvidos (STD) e de condutividade elétrica foram maiores nos tratamentos com morte por congelamento e pelo diquat e em mesocosmos colonizados sem controle da macrófita. Esse efeito pode ser devido à presença de material orgânico em suspensão e à maior concentração de nutrientes presentes na água. Comparando os mesocosmos sem plantas, sem e com a aplicação de diquat na superficie da água, os valores das características avaliadas foram estatisticamente similares, levando à conclusão de que as alterações observadas nos fatores analisados decorrem principalmente da decomposição das plantas.
\end{abstract}

Palavras-chave: aguapé, diquat, controle químico, impacto ambiental.

ABSTRACT - Besides their great importance in the aquatic environment dynamics, with the formation of dense colonization, the macrophytes cause several damages to the ecological equilibrium and multiple use of water. Under these conditions, control measures are required aiming to reduce the size of macrophyte populations. Among the most important aquatic weeds, the waterhyacinth (Eichhornia crassipes) is considered a major problem, its control being necessary in several sites in the world. The herbicide diquat has been used for waterhyacinth control due to its low cost, high efficacy, quickness of action and environmental safety. Aiming to evaluate the effects of waterhyacinth control and of diquat use on some water characteristics, an experiment was carried out under mesocosmic conditions, analyzing the following experimental situations: $\mathrm{CPCH}$ - mesocosm

1 Recebido para publicação em 7.2.2005 e na forma revisada em 27.3.2005.

Parte da dissertação de mestrado do primeiro autor.

2 Aluno do curso de pós-graduação em Produção Vegetal - FCAV/UNESP, Av. Libero Badaró, 478, 14870-540 Jaboticabal-SP, <atrevizoli@uol.com.br>; ${ }^{3}$ Professor Titular, Dep. de Biologia Aplicada à Agropecuária, FCAV/UNESP. 
colonized by waterhyacinth controlled by diquat spraying at $7.0 \mathrm{~L} \mathrm{ha-1}$ of Reward $\mathrm{R}$; CPCG mesocosm colonized by waterhyacinth destroyed by freezing; CPSH - mesocosm colonized by waterhyacinth, without control; SPCH-mesocosm without aquatic weeds and diquat spray on the water surface, and SPSH - mesocosm without aquatic weeds. Water temperature was higher in the mesocosms without waterhyacinth colonization due to the radiation penetration in the water column. The dissolved oxygen concentrations were lower in the mesocosms colonized by waterhyacinth and were reduced just after the weed control, due to the elevation of biological oxygen demand (BOD) during plant decomposition. The water $\mathrm{pH}$ was higher in the mesocosms without waterhyacinth colonization. The values of total dissolved solids (STD) and electric conductivity were higher in the mesocosm with waterhyacinth, regardless of weed control. Comparison of the mesocosm without macrophytes, with and without diquat application, showed that the values of the characteristics evaluated were similar, leading to the conclusion that the changes observed were due to plant decomposition.

Palavras-chave: Eichhornia crassipes, diquat, chemical control, environmental impact.

\section{INTRODUÇÃO}

O aguapé (Eichhornia crassipes) atualmente é uma planta cosmopolita. Sua origem é a América do Sul, mas, devido à beleza da planta e, especialmente, das flores, foi rapidamente introduzida em diferentes regiões do mundo, onde acabou se tornando a pior planta invasora de ambientes aquáticos (Holm et al., 1969). Esse caráter invasor se deve à sua alta capacidade de multiplicação vegetativa, fazendo com que grandes extensões sejam ocupadas rapidamente (Holm et al., 1969; Stewart, 1970; Lareo, 1981; Raju \& Reddy, 1988; Guitiérrez et al., 1996). Altas colonizações de aguapé trazem impactos negativos na economia pesqueira, devido principalmente às baixas concentrações de oxigênio dissolvido na coluna d'água; favorecem a proliferação de insetos, larvas e moluscos transmissores de doenças como encefalite, malária e esquistossomose; influenciam a redução do fluxo de água em canais, represas e rios; dificultam a navegação e a pesca; e são responsáveis por elevadas perdas de água por evapotranspiração (Weert \& Kamerling, 1974; Lallana et al., 1987; Velini, 2000).

A baixa diversidade e densidade de inimigos naturais e a eutrofização do ambiente são as duas principais causas do excessivo crescimento de plantas aquáticas (Esteves, 1998). Nessas condições, há necessidade do controle populacional dessas plantas para manter as colonizações dentro de limites em que seus efeitos benéficos ao ambiente aquático suplantem os efeitos prejudiciais. Esse controle pode ser feito pelo aumento de pressão de inimigos naturais (controle biológico) ou pela promoção da morte (controle químico) ou retirada das plantas do corpo hídrico (controle mecânico).

Em termos mundiais, o controle químico vem sendo utilizado com maior freqüência no controle das plantas daninhas aquáticas, principalmente devido à facilidade de aplicação (Velini, 2000), à eficiência de controle (Wojtalik et al., 1971; Boyle, 1980; Ahmed et al., 1980, 1982; Fairchild et al., 1998) e ao baixo custo (Blackburn \& Sutton, 1970). No Brasil, apesar de não haver nenhum herbicida registrado para o controle do aguapé, há vários estudos acadêmicos que comprovam a eficácia do controle químico (Gelmini, 1995; Bisigato, 1999; Martins, 2001; Neves et al., 2002; Martins et al., 2002). Dentre os herbicidas mais utilizados no exterior destaca-se o diquat, pelo seu baixo custo, rapidez de ação e segurança ambiental. É um herbicida muito usado no controle de plantas daninhas aquáticas nos Estados Unidos, sendo $60 \%$ do total aplicado no estado da Flórida, que utiliza cerca de 49.896 L por ano do produto formulado (Ritter et al., 2000).

Wellborn et al. (1969), Wilson \& Bond (1969), Couch \& Nelson (1982), Scott et al. (1984), Draxl et al. (1991), Fleckner (1991), Melendez et al. (1993) e Peterson et al. (1997) consideram o diquat um produto com risco de intoxicação para certos organismos aquáticos. No entanto, Joyce (1993) e Ritter et al. (2000) ponderam que o diquat é um produto seguro, 
pelo fato de dissipar-se rapidamente na água e não causar efeitos crônicos aos animais que ali vivem. Newman \& Way (1966) e El-Deen \& Rogers (1992) demonstraram que os efeitos mais marcantes são as conseqüências secundárias da aplicação do diquat em áreas com macrófitas.

Nesses estudos foram quantificados decréscimos da concentração do oxigênio dissolvido e altos valores de amônia, nitrito e fosfato, que influenciavam o crescimento de algas, zooplâncton, peixes e anfibios. Lugo et al. (1998) comprovaram que, na prática, os efeitos deletérios do controle do aguapé no zooplâncton ocorreram em virtude da redução na concentração de oxigênio dissolvido, provocada pela morte da planta, e não diretamente pelos herbicidas diquat e 2,4-D, utilizados na aplicação.

Diante dos prováveis riscos que o controle com herbicidas pode oferecer, objetivou-se com este trabalho avaliar isoladamente os possíveis impactos causados pela aplicação de diferentes manejos, principalmente com o uso de diquat, no controle de Eichhornia crassipes, em alguns parâmetros físicos de qualidade da água em condições de mesocosmos.

\section{MATERIAL E MÉTODOS}

O presente experimento foi conduzido na área experimental do Laboratório Giorgio De Marinis, do Departamento de Biologia Aplicada à Agropecuária da Faculdade de Ciências Agrárias e Veterinárias, UNESP, campus de Jaboticabal.

Os estudos foram realizados em 20 mesocosmos, constituídos por tanques de concreto com uma área de $2,71 \mathrm{~m}^{2}$ e $0,40 \mathrm{~m}$ de profundidade, com volume de $1.080 \mathrm{~L}$. A entrada e a saída da água dos mesocosmos ocorreram pela superfície. A água que abastece os mesocosmos é proveniente de um poço cisterna, sendo fornecida em quantidade suficiente para que haja um Tempo de Residência Hidráulico (TRH) de sete dias. Ao deixar os mesocosmos, a água passa por um sistema de carvão ativado, antes de ser descartada no sistema de esgoto.

O substrato utilizado como sedimento de fundo do mesocosmo foi composto de areia, solo de barranco e torta de filtro, nas mesmas proporções volumétricas. Em cada mesocosmo foi colocada uma camada de $10 \mathrm{~cm}$ de sedimento. Os indivíduos de aguapé foram coletados em lagoa marginal do rio Mogi-Guaçu, no município de Barrinha-SP.

Os tratamentos estudados constaram de cinco situações de manejo do mesocosmo, a saber:

- CPCG - mesocosmo colonizado por macrófitas aquáticas, as quais foram mortas por congelamento.

- $\mathbf{C P C H}$ - mesocosmo colonizado por macrófitas aquáticas, as quais foram controladas pela aplicação do herbicida diquat, na formulação Reward ${ }^{\circledR}\left(7 \mathrm{~L} \mathrm{ha}^{-1}\right)$.

- CPSH - mesocosmo colonizado por macrófitas aquáticas sem qualquer controle.

- $\mathbf{S P C H}$ - mesocosmo mantido sem macrófitas aquáticas e com aplicação de diquat, formulação $\operatorname{Reward}^{\circledR}\left(7 \mathrm{~L} \mathrm{ha}^{-1}\right)$, diretamente sobre a lâmina d'água.

- SPSH - mesocosmo mantido sem macrófitas aquáticas e sem aplicação de herbicidas.

A distribuição dos tratamentos nos mesocosmos obedeceu ao delineamento inteiramente casualizado, com quatro repetições.

A aplicação do herbicida ocorreu no dia 15.8.2003, das 18 h30 às 19 h05. O herbicida diquat foi usado na formulação comercial Reward $\AA$, que contém $200 \mathrm{~g} \mathrm{~L}^{-1}$ de ingrediente ativo. A dose da formulação comercial utilizada foi de $7,0 \mathrm{~L} \mathrm{ha}^{-1}$. Esta dose está no limite superior da faixa de recomendações do produto para controle de aguapé - condição recomendada para estudos de impacto ambiental (pior cenário). Na calda do herbicida foi adicionado o adjuvante Agral, na concentração de $50 \mathrm{~mL} 100 \mathrm{~L}^{-1}$. A aplicação foi feita com pulverizador costal à pressão constante, munido de bicos 11002 XR VK, numa pressão de 25 p.s.i., que proporcionou um consumo de calda equivalente a $200 \mathrm{~L} \mathrm{ha}^{-1}$. Por ocasião da aplicação do diquat as condições climáticas locais foram: temperatura ambiente de $20{ }^{\circ} \mathrm{C}$, umidade relativa do ar de $66 \%$ e sem vento.

Para evitar que a deriva da calda do herbicida atingisse outros mesocosmos, a barra do pulverizador foi conduzida dentro de uma

Planta Daninha, Viçosa-MG, v. 23, n. 2, p. 233-242, 2005 
estrutura de metal revestida por plástico, que protegia o fundo e as laterais da área aplicada.

A morte das macrófitas por congelamento foi efetuada gradualmente, seguindo as seguintes datas: 15.8.2003, 22.8.2003 e 1.9.2003. A cada um destes dias, um terço das macrófitas do mesocosmo foi retirado, pesado e colocado em freezer na temperatura de $-4{ }^{\circ} \mathrm{C}$ por 48 horas, para depois ser retornado a seus respectivos mesocosmos. Antes de as plantas mortas serem recolocadas em seus respectivos mesocosmos, foi esperado que elas voltassem à temperatura ambiente.

Durante 63 dias foram efetuadas leituras dos indicadores de qualidade da água na profundidade entre 10 e $15 \mathrm{~cm}$, no horário entre 14 e 14h30. Essa profundidade foi estabelecida para evitar que o sedimento fosse perturbado com o movimento da sonda, alterando os dados coletados. As avaliações foram realizadas com sonda YSI 556 MPS da Yellowspring Co, determinando as seguintes características: temperatura $\left({ }^{\circ} \mathrm{C}\right)$, concentração de oxigênio dissolvido $\left(\mathrm{mg} \mathrm{L}^{-1}\right), \mathrm{pH}$, sólidos totais dissolvidos (STD) $\left(\mathrm{g} \mathrm{L}^{-1}\right)$ e condutividade elétrica $\left(\mathrm{mS} \mathrm{cm}^{-1}\right)$. A partir dos dados diários coletados, foram feitas médias semanais, totalizando nove semanas. A semana anterior à aplicação é representada pelo número 0 , e as oito semanas restantes referem-se aos dados coletados após a aplicação.

Todos os valores obtidos foram submetidos a testes de análise de variância e teste de Tukey, utilizando o programa estatístico SAS versão 8.0.

\section{RESULTADOS E DISCUSSÃO}

\section{Temperatura}

Os valores relativos à temperatura da água determinada no horário das 14 às 14 h30 estão apresentados na Tabela 1. Esse é um período de elevada atividade fotossintética dos organismos presentes na água. Houve interação entre o período de avaliação (semana) e a condição de manejo das macrófitas no mesocosmo (tratamento). Ao longo do período experimental, a temperatura média semanal da água aumentou em todos os tratamentos, pois o início do experimento ocorreu em agosto e se estendeu até novembro - período que abrange o final do inverno até meados da primavera.

Nas condições sem colonização com o aguapé, a temperatura da água dos mesocosmos foi superior à dos colonizados, mesmo naqueles em que houve controle do aguapé. A cobertura das plantas vivas de aguapé promove proteção substancial da coluna d'água contra os raios solares e o aumento da temperatura, pois, além da densa barreira física proporcionada, as plantas absorvem os raios solares e o transformam em energia química por meio do processo fotossintético, não permitindo a formação de radiação calorífica.

Outro fato observado e importante a ser destacado é o crescimento de populações de Salvinia molesta nos mesocosmos em que o aguapé foi controlado tanto pelo diquat quanto pelo congelamento. Apesar de todo o cuidado para que apenas plantas de aguapé fossem

Tabela 1 - Valores médios semanais de temperatura $\left({ }^{\circ} \mathrm{C}\right)$ da água em mesocosmos submetidos a diferentes manejos de colonização por Eichhornia crassipes e aplicação de diquat

\begin{tabular}{|c|c|c|c|c|c|c|c|c|c|c|c|}
\hline \multirow{3}{*}{ Tratamento } & \multicolumn{9}{|c|}{ Semana após aplicação } & \multirow{3}{*}{ F } & \multirow{3}{*}{$\mathrm{CV} \%$} \\
\hline & \multicolumn{9}{|c|}{$\left({ }^{\circ} \mathrm{C}\right)$} & & \\
\hline & 0 & 1 & 2 & 3 & 4 & 5 & 6 & 7 & 8 & & \\
\hline CPCG & $20,83 \mathrm{ABbcd}$ & $18,91 \mathrm{Bd}$ & $20,03 \mathrm{Bcd}$ & $21,82 \mathrm{~B} \mathrm{bc}$ & $22,19 \mathrm{~B} \mathrm{bc}$ & $22,44 \mathrm{~B} \mathrm{~b}$ & $24,79 \mathrm{Ba}$ & $25,30 \mathrm{Ba}$ & $25,67 \mathrm{Ba}$ & $23,71^{* *}$ & 4,36 \\
\hline $\mathrm{CPCH}$ & 19,53 Bef & $18,87 \mathrm{Bf}$ & $20,01 \mathrm{Be}$ & $21,45 \mathrm{BCd}$ & $22,24 \mathrm{Bc}$ & $22,19 \mathrm{BCc}$ & $24,66 \mathrm{Bb}$ & $24,90 \mathrm{BCab}$ & $25,40 \mathrm{BCa}$ & $281,94 * *$ & 1,30 \\
\hline CPSH & $19,38 \mathrm{Be}$ & $18,62 \mathrm{Bf}$ & $19,56 \mathrm{Bc}$ & $21,08 \mathrm{Cd}$ & $21,91 \mathrm{Bc}$ & $21,85 \mathrm{Cc}$ & $24,28 \mathrm{Bb}$ & $24,59 \mathrm{Cb}$ & $25,14 \mathrm{Ca}$ & $724,36^{* *}$ & 0,82 \\
\hline SPCH & $23,33 \mathrm{Ac}$ & $23,33 \mathrm{Ac}$ & $23,77 \mathrm{Ac}$ & $25,89 \mathrm{Ab}$ & $25,42 \mathrm{Ab}$ & $26,26 \mathrm{Ab}$ & $29,10 \mathrm{Aa}$ & $29,03 \mathrm{Aa}$ & $28,30 \mathrm{Aa}$ & $169,83^{* *}$ & 1,37 \\
\hline SPSH & $23,22 \mathrm{Ad}$ & $23,40 \mathrm{Ad}$ & $23,72 \mathrm{Ad}$ & $25,90 \mathrm{Ac}$ & $25,46 \mathrm{Ac}$ & $26,19 \mathrm{Ac}$ & $28,98 \mathrm{Ab}$ & $29,12 \mathrm{Aa}$ & $28,14 \mathrm{Ab}$ & $192,21 * *$ & 1,29 \\
\hline$F$ & $8,8^{* *}$ & $123,89^{* *}$ & $304,54 * *$ & $228,37 * *$ & $235,32 * *$ & $315,43 * *$ & $382,75^{* *}$ & $265,14 * *$ & $200,43 * *$ & & \\
\hline CV\% & 6,11 & 2,18 & 1,14 & 1,39 & 1,01 & 1,05 & 0,94 & 1,05 & 0,82 & & \\
\hline
\end{tabular}

** significativo a $1 \%$ de probabilidade, * significativo a $5 \%$ de probabilidade e ${ }^{\mathrm{ns}}$ não-significativo.

Médias seguidas por mesma letra minúscula na linha e maiúscula na coluna não diferem entre si pelo teste de Tukey a 5\% de probabilidade. 
colocadas nos mesocosmos, é possível que em suas raízes houvesse pequenos propágulos da salvínia, que não foram percebidos na montagem do experimento. Nessas duas condições de manejo dos mesocosmos, a salvínia cresceu vigorosamente a partir da terceira semana e manteve o efeito parcial de cobertura. Esta possivelmente é uma explicação do motivo pelo qual os mesocosmos com cobertura de macrófitas vivas diferiram tão pouco daqueles nos quais o aguapé foi morto.

É importante salientar que os efeitos da cobertura de macrófitas aquáticas sobre a amortização da temperatura da água já foram observados por inúmeros autores. No Brasil, destaca-se o trabalho de Guimarães et al. (2003), os quais observaram resultados similares estudando os impactos do controle de colonização mista de macrófitas aquáticas, também em condições de mesocosmos. Utilizando o herbicida 2,4-D, a população de Salvinia herzogii não foi controlada e colonizou toda a superficie da água, substituindo as plantas de aguapé.

\section{Oxigênio dissolvido}

Na Tabela 2 estão apresentados os resultados referentes às medidas dos teores de oxigênio dissolvido na água, nos mesocosmos, no horário das 14 às 14 h30.

Com o desenvolvimento do período experimental, apenas houve elevação dos teores de oxigênio dissolvido nas duas condições em que os mesocosmos não foram colonizados por macrófitas. Nos mesocosmos colonizados por aguapé, independentemente do controle, as concentrações foram muito baixas, sempre estatisticamente inferiores às daqueles sem colonização, atingindo valores abaixo de 5,0 mg L ${ }^{-1}$, considerado limite por Brasil (1986), na Resolução CONAMA n ${ }^{\circ} 20$.

Comparando os dois mesocosmos sem colonização de aguapé, em apenas uma semana, a concentração de oxigênio dissolvido foi inferior naqueles que receberam o diquat. Como esse comportamento estatístico ocorreu na quarta semana após aplicação, o menor valor pode ser decorrente do acaso ou da ação de algum produto de degradação do diquat que tenha afetado a produção ou a disponibilidade de oxigênio livre na coluna d'água.

Dos três mesocosmos colonizados por aguapé, não houve diferença estatisticamente significativa nas três condições de manejo: controle por congelamento, controle por diquat e sem controle. Nas três condições, há algumas razões coincidentes e algumas diversas para explicar os baixos teores de oxigênio dissolvido na água. Em todas essas condições há a barreira física da cobertura vegetal à penetração da radiação solar, prejudicando a fotossintese da coluna d'água (Neves et al., 2002). Esse efeito provavelmente foi muito mais drástico nos mesocosmos em que as plantas de aguapé não foram mortas. Ainda nos mesocosmos com as plantas vivas, há demanda respiratória de oxigênio pelo denso sistema radicular da macrófita e dos organismos perifiticos associados (Neves et al., 2002).

Tabela 2 - Valores médios semanais de oxigênio dissolvido $\left(\mathrm{mg} \mathrm{L}^{-1}\right)$ da água em mesocosmos submetidos a diferentes manejos de colonização por Eichhornia crassipes e aplicação de diquat

\begin{tabular}{|c|c|c|c|c|c|c|c|c|c|c|c|}
\hline \multirow{3}{*}{ Tratamento } & \multicolumn{9}{|c|}{ Semana após aplicação } & \multirow{3}{*}{$\mathrm{F}$} & \multirow{3}{*}{$\mathrm{CV} \%$} \\
\hline & \multicolumn{9}{|c|}{$\left(\mathrm{mg} \mathrm{L}^{-1}\right)$} & & \\
\hline & 0 & 1 & 2 & 3 & 4 & 5 & 6 & 7 & 8 & & \\
\hline CPCG & $2,25 \mathrm{Ba}$ & $1,80 \mathrm{Bab}$ & 1,69 Bb & $1,35 \mathrm{Bbcd}$ & $0,84 \mathrm{Cd}$ & $0,96 \mathrm{Bcd}$ & $1,04 \mathrm{Bcd}$ & $1,26 \mathrm{Bbcd}$ & $1,43 \mathrm{Bbc}$ & $14,89 * *$ & 16,59 \\
\hline $\mathrm{CPCH}$ & $2,36 \mathrm{Ba}$ & $2,35 \mathrm{Ba}$ & $1,17 \mathrm{Bb}$ & $0,99 \mathrm{Bb}$ & $0,85 \mathrm{Cb}$ & $1,08 \mathrm{Bb}$ & $1,02 \mathrm{Bb}$ & $1,22 \mathrm{Bb}$ & $1,36 \mathrm{Bb}$ & $18,68^{* *}$ & 19,09 \\
\hline CPSH & $2,55 \mathrm{Babc}$ & $3,12 \mathrm{Ba}$ & $2,73 \mathrm{Ba}$ & 2,33 Babc & $1,69 \mathrm{Cc}$ & $1,92 \mathrm{Bbc}$ & $1,63 \mathrm{Bc}$ & $1,79 \mathrm{Bbc}$ & $1,91 \mathrm{Bbc}$ & $19,82^{* *}$ & 19,82 \\
\hline SPCH & $9,56 \mathrm{Ab}$ & $9,36 \mathrm{Ab}$ & $10,13 \mathrm{Aab}$ & $10,95 \mathrm{Aab}$ & $9,93 \mathrm{Bab}$ & $12,40 \mathrm{Aab}$ & $13,00 \mathrm{Aab}$ & $13,75 \mathrm{Aa}$ & $11,33 \mathrm{Aab}$ & $3,28^{* *}$ & 15,66 \\
\hline SPSH & $9,92 \mathrm{Aab}$ & $9,62 \mathrm{Ab}$ & $10,92 \mathrm{Aab}$ & $12,38 \mathrm{Aab}$ & $12,20 \mathrm{Aab}$ & $15,54 \mathrm{Aab}$ & $17,01 \mathrm{Aa}$ & $13,22 \mathrm{Aab}$ & $15,00 \mathrm{Aab}$ & $2,85^{*}$ & 23,62 \\
\hline$F$ & $201,08^{* *}$ & $98,74 * *$ & $68,47^{* *}$ & $163,00^{* *}$ & $53,29 * *$ & $34,29^{* *}$ & $39,87^{* *}$ & $41,64 * *$ & $48,39^{* * *}$ & & \\
\hline $\mathrm{CV} \%$ & 10,65 & 14,98 & 21,69 & 15,59 & 11,09 & 37,57 & 36,06 & 32,79 & 30,03 & & \\
\hline
\end{tabular}

** significativo a $1 \%$ de probabilidade, * significativo a $5 \%$ de probabilidade e ${ }^{\text {ns }}$ não-significativo.

Médias seguidas por mesma letra minúscula na linha e maiúscula na coluna não diferem entre si pelo teste de Tukey a 5\% de probabilidade. 
Nos mesocosmos em que as plantas foram mortas, por congelamento ou pelo controle químico, a interceptação da radiação tendeu a decrescer com o tempo, porém houve incremento da demanda biológica de oxigênio (DBO) em decorrência da degradação das plantas mortas. Com a degradação mais avançada, houve liberação de nutrientes minerais, o que permitiu o crescimento populacional de salvínia. Esta macrófita secundária (no caso do presente estudo) passou a interceptar os raios solares e consumir oxigênio da água pela sua respiração radicular.

Outro ponto a ser destacado é que as macrófitas, tanto o aguapé como a salvínia, e os resíduos vegetais flutuantes reduzem a superficie de troca de oxigênio da interface águaatmosfera.

É interessante destacar que em alguns mesocosmos sem macrófitas e sem aplicação do diquat ocorreram alguns rápidos blooms de algas, os quais, pelo horário da avaliação, alcançaram alguns valores bastante altos nas concentrações de oxigênio dissolvido na água. Schwegler (1978) havia observado que, nessas condições, as concentrações de oxigênio poderiam atingir valores entre 15 e $20 \mathrm{mg} \mathrm{L}^{-1}$, muito similares aos observados na presente pesquisa.

Com relação a esta característica de qualidade da água, oxigênio dissolvido, é importante ressaltar que a presença das plantas vivas de aguapé ou da condição de morte, decomposição e substituição das populações de plantas flutuantes manteve as concentrações na água abaixo de niveis críticos para a ictiofauna. Os menores valores foram observados entre a terceira e a quarta semana nos mesocosmos com controle do aguapé. Nos mesocosmos sem colonização, a aplicação do diquat não afetou os teores de oxigênio dissolvido, provavelmente por sua rápida dissipação em águas rasas e transparentes, sob insolação direta (Joyce, 1993; Ritter et al., 2000), como ocorreu no desenvolvimento do ensaio. Outro fato importante de ser destacado é que nos mesocosmos colonizados (com ou sem controle do aguapé) houve uniformidade na concentração de oxigênio dissolvido ao longo do período experimental, sendo anulado o crescimento dos valores observados nos mesocosmos sem plantas aquáticas, ao longo do período experimental.

\section{pH}

Na Tabela 3 estão apresentados os resultados referentes às medidas dos valores de $\mathrm{pH}$ da água nos mesocosmos no horário das 14 às 14 h30.

Nos mesocosmos sem a colonização de aguapé, o pH da água foi estatisticamente similar em praticamente todas as semanas avaliadas, com exceção da quarta semana, em que os valores dos mesocosmos que receberam o herbicida foram menores que os daqueles sem aplicação do produto. Esses resultados sugerem que a aplicação do diquat não altera o potencial hidrogênio iônico da água.

Tabela 3 - Valores médios semanais de $\mathrm{pH}$ da água em mesocosmos submetidos a diferentes manejos de colonização por Eichhornia crassipes e aplicação de diquat

\begin{tabular}{|c|c|c|c|c|c|c|c|c|c|c|c|}
\hline \multirow{3}{*}{ Tratamento } & \multicolumn{9}{|c|}{ Semana após aplicação } & \multirow{3}{*}{$\mathrm{F}$} & \multirow{3}{*}{$\mathrm{CV} \%$} \\
\hline & \multicolumn{9}{|c|}{$\left(\mathrm{CaCl}_{2}\right)$} & & \\
\hline & 0 & 1 & 2 & 3 & 4 & 5 & 6 & 7 & 8 & & \\
\hline CPCG & 6,36 Bde & 6,51 BCcde & $6,82 \mathrm{BCab}$ & $6,64 \mathrm{BCbc}$ & $6,99 \mathrm{BCa}$ & $6,85 \mathrm{Ba}$ & $6,56 \mathrm{Bcd}$ & $6,35 \mathrm{Be}$ & 6,38 Bde & $30,26 * *$ & 1,3 \\
\hline $\mathrm{CPCH}$ & $6,23 \mathrm{Be}$ & $6,22 \mathrm{Ce}$ & $6,66 \mathrm{BCb}$ & $6,60 \mathrm{BCbc}$ & $6,98 \mathrm{BCa}$ & $6,85 \mathrm{Ba}$ & $6,66 \mathrm{Bb}$ & $6,47 \mathrm{Bcd}$ & $6,45 \mathrm{Bd}$ & $79,28 * *$ & 0,87 \\
\hline CPSH & $6,28 \mathrm{Be}$ & "'6,31 Cde & 6,56 Cbcd & 6,39 Ccde & $6,82 \mathrm{Ca}$ & 6,67 Bab & $6,56 \mathrm{Bbcd}$ & $6,63 \mathrm{Babc}$ & $6,60 \mathrm{Babc}$ & $10,93 * *$ & 1,65 \\
\hline $\mathrm{SPCH}$ & $6,82 \mathrm{Ac}$ & $6,89 \mathrm{ABc}$ & $7,24 \mathrm{ABc}$ & $7,27 \mathrm{ABc}$ & $7,56 \mathrm{Bbc}$ & $8,85 \mathrm{Ab}$ & $8,98 \mathrm{Ab}$ & $9,89 \mathrm{Aa}$ & $9,47 \mathrm{Aa}$ & $40,74 * *$ & 4,58 \\
\hline SPSH & $6,75 \mathrm{Ac}$ & $6,95 \mathrm{Ac}$ & 7,58 Abc & 7,78 Abc & 8,46 Aabc & 9,23 Aab & 9,39 Aab & 9,34 Aab & $9,68 \mathrm{Aa}$ & $8,55^{* *}$ & 9,17 \\
\hline $\mathrm{F}$ & $33,89 *$ & 14,04 & $7,85 * *$ & $11,00 * *$ & $20,09 * *$ & $33,20 * *$ & $33,63 * *$ & $42,91 * *$ & $39,37 * *$ & & \\
\hline $\mathrm{CV} \%$ & 1,45 & 2,67 & 4,4 & 5,01 & 4,09 & 5,6 & 6,45 & 6,81 & 7,02 & & \\
\hline
\end{tabular}

** significativo a $1 \%$ de probabilidade, * significativo a $5 \%$ de probabilidade e ${ }^{\text {ns }}$ não-significativo.

Médias seguidas por mesma letra minúscula na linha e maiúscula na coluna não diferem entre si pelo teste de Tukey a 5\% de probabilidade. 
Todos os mesocosmos colonizados com aguapé apresentaram valores de $\mathrm{pH}$ da água estatisticamente inferiores aos daqueles nãocolonizados. Segundo Esteves (1998), algas e macrófitas submersas podem elevar o $\mathrm{pH}$ da água pela assimilação do $\mathrm{CO}_{2}$ no processo fotossintético, especialmente em águas com baixa capacidade de neutralizar ácidos, ou seja, águas com baixa alcalinidade. $\mathrm{O}$ autor comenta ainda que a atividade heterotrófica pode promover redução do $\mathrm{pH}$ da água. Considerando que a parte submersa das plantas de aguapé é o sistema radicular, o efeito sobre o pH da água é o de redução dos valores, em decorrência da atividade respiratória intensa do grande volume de raízes que esta planta apresenta.

Nas três condições de mesocosmos colonizados, com e sem controle do aguapé, os valores do $\mathrm{pH}$ da água foram estatisticamente similares. É possivel que, no mesocosmo no qual a macrófita foi mantida sem controle, a intensa atividade respiratória do sistema radicular na produção do gás carbônico e a interceptação da luz solar que impedia a atividade fotossintética tenham determinado o baixo valor de $\mathrm{pH}$. Nos mesocosmos onde a planta foi morta, tanto pelo congelamento como pelo herbicida, a interceptação da radiação solar não foi muito intensa no início (antes da mudança de flora pela salvínia), mas houve intensa atividade heterotrófica na decomposição dos resíduos da planta, anulando parcialmente o efeito positivo do processo fotossintético no $\mathrm{pH}$.

\section{Sólidos totais dissolvidos}

Na Tabela 4 estão apresentados os resultados referentes às medidas dos valores de sólidos totais dissolvidos (STD) da água, nos mesocosmos, no horário das 14 às 14 h30.

Os menores valores de STD sempre foram observados nos mesocosmos sem plantas, com ou sem aplicação do diquat. O crescimento das algas microscópicas não foi suficiente para proporcionar material em quantidade que promovesse aumento no valor medido da STD.

Nos mesocosmos com controle do aguapé houve rápido incremento dos sólidos dissolvidos totais, em decorrência da quebra das estruturas de contingenciamento do conteúdo celular e de sua liberação para a coluna d'água. Esse incremento ocorreu até a quarta e quinta semanas para os mesocosmos com morte por congelamento e por diquat, respectivamente. Após esse período houve redução dos valores de STD, uma vez que o processo de decomposição estava avançado e praticamente restavam as estruturas de difícil degradação, como celulose, hemicelulose e lignina.

A partir da quarta semana após o início das amostragens, houve incremento de STD nas parcelas colonizadas por aguapé e sem controle. A possível explicação para esse comportamento é o adensamento de morte de plantas por competição, pois o crescimento da macrófita foi muito intenso. Com a morte de partes das plantas, houve bastante disponibilidade de material passivel de decomposição, influenciando positivamente o valor da concentração de sólidos dissolvidos totais.

\section{Condutividade elétrica}

Na Tabela 5 encontram-se os resultados referentes às medidas dos valores de condutividade elétrica da água nos mesocosmos no horário das 14 às 14h30. Da segunda à quarta semana após o controle, os mesocosmos com morte por congelamento ou por aplicação de diquat apresentaram valores de condutividade elétrica superiores aos daqueles colonizados por aguapé e sem controle e daqueles sem colonização, com e sem aplicação do herbicida. É importante destacar que o aumento da condutividade elétrica, com grande probabilidade, é decorrente da liberação de nutrientes durante o processo de decomposição das plantas. $\mathrm{Na}$ primeira semana após o controle, apenas os mesocosmos em que as plantas foram mortas por congelamento apresentaram valores superiores aos demais, provavelmente porque o processo de congelamento promove o rompimento das paredes celulares e ocorre o rápido extravasamento do conteúdo celular.

$\mathrm{Na}$ metade final do período experimental ocorreram incrementos dos valores da condutividade elétrica para os mesocosmos sem colonização de aguapé e para aqueles colonizados e sem controle. Aparentemente, esse aumento está relacionado à degradação de material orgânico presente no sedimento,

Planta Daninha, Viçosa-MG, v. 23, n. 2, p. 233-242, 2005 
Tabela 4 - Valores médios semanais de STD $\left(\mathrm{g} \mathrm{L}^{-1}\right)$ da água em mesocosmos submetidos a diferentes manejos de colonização por Eichhornia crassipes e aplicação de diquat

\begin{tabular}{|c|c|c|c|c|c|c|c|c|c|c|c|}
\hline \multirow{3}{*}{ Tratamento } & \multicolumn{9}{|c|}{ Semana após aplicação } & \multirow{3}{*}{$F$} & \multirow{3}{*}{$\mathrm{CV} \%$} \\
\hline & \multicolumn{9}{|c|}{$\left(\mathrm{g} \mathrm{L}^{-1}\right)$} & & \\
\hline & 0 & 1 & 2 & 3 & 4 & 5 & 6 & 7 & 8 & & \\
\hline CPCG & $0,115 \mathrm{Af}$ & 0,142 Ade & $0,157 \mathrm{Acd}$ & $0,172 \mathrm{Abc}$ & $0,222 \mathrm{Aa}$ & $0,182 \mathrm{Bb}$ & $0,130 \mathrm{Bef}$ & $0,127 \mathrm{BCef}$ & $0,127 \mathrm{Bef}$ & $48,78^{* *}$ & 6,44 \\
\hline $\mathrm{CPCH}$ & $0,107 \mathrm{Ae}$ & $0,125 \mathrm{Bde}$ & $0,155 \mathrm{Abc}$ & $0,160 \mathrm{Ab}$ & $0,207 \mathrm{Aa}$ & $0,205 \mathrm{Aa}$ & $0,157 \mathrm{Abc}$ & $0,145 \mathrm{ABbcd}$ & $0,137 \mathrm{Bcd}$ & $51,93 * *$ & 5,93 \\
\hline CPSH & $0,117 \mathrm{Ac}$ & $0,117 \mathrm{BCc}$ & $0,107 \mathrm{Bc}$ & $0,117 \mathrm{Bc}$ & $0,160 \mathrm{Bab}$ & $0,177 \mathrm{Ba}$ & $0,155 \mathrm{Ab}$ & $0,165 \mathrm{Aab}$ & $0,165 \mathrm{Aab}$ & $41,76^{* *}$ & 5,84 \\
\hline SPCH & $0,110 \mathrm{Aab}$ & $0,117 \mathrm{BCa}$ & $0,100 \mathrm{Ba}$ & $0,105 \mathrm{Bab}$ & $0,105 \mathrm{Cab}$ & $0,107 \mathrm{Cab}$ & $0,110 \mathrm{Cab}$ & $0,120 \mathrm{BCa}$ & $0,112 \mathrm{Cab}$ & $3,01 *$ & 6,62 \\
\hline SPSH & $0,110 \mathrm{Aabc}$ & $0,105 \mathrm{Cabc}$ & $0,097 \mathrm{Bc}$ & $0,112 \mathrm{Babc}$ & $0,102 \mathrm{Cbc}$ & $0,110 \mathrm{Cabc}$ & $0,117 \mathrm{Cab}$ & $0,117 \mathrm{Cab}$ & $0,122 \mathrm{BCa}$ & $4,44 * *$ & 6,85 \\
\hline$F$ & $2,25^{\mathrm{ns}}$ & $18,18 * *$ & $31,59^{* *}$ & $77,22^{* *}$ & $141,42^{* *}$ & $130,26^{* *}$ & $61,92^{* *}$ & 11,08 & $17,20 * *$ & & \\
\hline CV\% & 4,89 & 5,31 & 8,68 & 5,2 & 5,89 & 5,01 & 4,08 & 8,86 & 7,26 & & \\
\hline
\end{tabular}

** significativo a $1 \%$ de probabilidade, $*$ significativo a $5 \%$ de probabilidade e ${ }^{\text {ns }}$ não-significativo.

Médias seguidas por mesma letra minúscula na linha e maiúscula na coluna não diferem entre si pelo teste de Tukey a 5\% de probabilidade.

Tabela 5 - Valores médios semanais de condutividade elétrica $\left(\mathrm{mS} \mathrm{cm}^{-1}\right)$ da água em mesocosmos submetidos a diferentes manejos de colonização por Eichhornia crassipes e aplicação de diquat

\begin{tabular}{|c|c|c|c|c|c|c|c|c|c|c|c|}
\hline \multirow{3}{*}{ Tratamento } & \multicolumn{9}{|c|}{ Semana após aplicação } & \multirow{3}{*}{$\mathrm{F}$} & \multirow{3}{*}{$\mathrm{CV} \%$} \\
\hline & \multicolumn{9}{|c|}{$\left(\mathrm{mS} \mathrm{cm}^{-1}\right)$} & & \\
\hline & 0 & 1 & 2 & 3 & 4 & 5 & 6 & 7 & 8 & & \\
\hline CPCG & $0,160 \mathrm{Ae}$ & 0,190 Acd & 0,217 Abc & $0,250 \mathrm{Aa}$ & $0,240 \mathrm{Aab}$ & $0,182 \mathrm{Bde}$ & $0,202 \mathrm{Bcd}$ & $0,197 \mathrm{Ccd}$ & $0,195 \mathrm{Bcd}$ & $21,31 * *$ & 5,96 \\
\hline $\mathrm{CPCH}$ & $0,150 \mathrm{Ac}$ & $0,167 \mathrm{Bc}$ & $0,212 \mathrm{Ab}$ & $0,232 \mathrm{Aab}$ & $0,215 \mathrm{Bab}$ & $0,207 \mathrm{Ab}$ & $0,242 \mathrm{Aa}$ & $0,227 \mathrm{Abab}$ & $0,210 \mathrm{Bb}$ & $26,09 * *$ & 5,68 \\
\hline CPSH & $0,155 \mathrm{Abc}$ & $0,157 \mathrm{Bbc}$ & $0,150 \mathrm{Bc}$ & $0,170 \mathrm{Bbc}$ & $0,162 \mathrm{Cbc}$ & $0,177 \mathrm{Bb}$ & $0,232 \mathrm{Aa}$ & $0,250 \mathrm{Aa}$ & $0,255 \mathrm{Aa}$ & $57,18^{* *}$ & 5,99 \\
\hline SPCH & $0,165 \mathrm{Ac}$ & $0,167 \mathrm{Bbc}$ & $0,150 \mathrm{Bc}$ & $0,167 \mathrm{Bbc}$ & $0,167 \mathrm{Cbc}$ & $0,167 \mathrm{Bbc}$ & $0,187 \mathrm{Bab}$ & $0,200 \mathrm{BCa}$ & $0,187 \mathrm{Bab}$ & $11,69 * *$ & 5,14 \\
\hline SPSH & $0,160 \mathrm{Ab}$ & $0,160 \mathrm{Bb}$ & $0,150 \mathrm{Bb}$ & $0,167 \mathrm{Bbc}$ & $0,162 \mathrm{Cbc}$ & $0,167 \mathrm{Bbc}$ & $0,192 \mathrm{Ba}$ & $0,197 \mathrm{Ca}$ & $0,197 \mathrm{Ba}$ & $13,34 * *$ & 5,73 \\
\hline $\mathrm{F}$ & $1,95^{\mathrm{ns}}$ & $9,62 * *$ & $29,9 * *$ & $68,82 * *$ & $50,58^{* *}$ & $13,79 * *$ & $26,40^{* *}$ & $11,77^{* *}$ & $13,85^{* *}$ & & \\
\hline $\mathrm{CV} \%$ & 5,16 & 4,9 & 7,41 & 4,93 & 5,32 & 4,9 & 4,52 & 6,39 & 6,93 & & \\
\hline
\end{tabular}

** significativo a $1 \%$ de probabilidade, * significativo a $5 \%$ de probabilidade e ${ }^{\mathrm{ns}}$ não-significativo.

Médias seguidas por mesma letra minúscula na linha e maiúscula na coluna não diferem entre si pelo teste de Tukey a $5 \%$ de probabilidade.

alterando paulatinamente o equilíbrio iônico da interface água e sedimento. Com esse tipo de tendência, na oitava semana houve similaridade estatística entre os valores observados para os dois mesocosmos com controle da macrófita e os dois sem colonização. Esse fato pode estar relacionado ao profuso crescimento de salvínia nos mesocosmos que haviam sido submetidos ao controle, mobilizando parte do nutriente dissociado na água.

Observa-se que as alterações nos valores das concentrações de oxigênio dissolvido e de sólidos dissolvidos totais e os valores de $\mathrm{pH}$ e de condutividade elétrica estão relacionados à morte e decomposição das plantas. A aplicação de diquat na superfície da água não promoveu alterações significativas nas características da água estudada. A cobertura de macrófitas sem controle contribuiu para redução do pH e da concentração de oxigênio dissolvido da água.

\section{LITERATURA CITADA}

AHMED, S. A.; ITO, M.; UEKI, K. Water quality as affected by waterhyacinth decomposition after autting or 2,4-D aplication. Weed Res. Japan, v. 27, p. 34-39, 1980.

AHMED, S. A.; ITO, M.; UEKI, K. Water quality as affected by waterhycinth decomposition after 2,4-D and ametryne application. Weed Res. Japan, v. 25, p. 286-293, 1982.

BISIGATO, A. E. F. Controle quimico de aguapé (Eichhornia crassipes (Mart.) Solms) e alface d'água (Pistia stratiotes L.), em duas formulações, aplicado nos horários diurno e noturno. 1999. $57 \mathrm{f}$. Monografia (Trabalho de Graduação em Agronomia) - Universidade Estadual Paulista, Jaboticabal, 1999. 
BLACKBURN, R. D.; SUTTON, D. L. The raise of herbicides in ponds, lakes and streams. In: FAO INTERNATIONAL CONFERENCE ON WEED CONTROL, 1., 1970, Davis. University of California. Proceedings... Davis: University of California, 1970. p. $374-386$

BOYDE, C. E. Water quality: an introduction: Boston: Kluwer Academic, 2000. 330 p.

BOYLE. T. P. Effects of the aquatic herbicide 2,4-D DMA on the ecology of experimental ponds. Environ. Poll. Series A, v. 21, p. 35-49, 1980.

BRASIL. Ministério do Meio Ambiente. Conselho Nacional do Meio Ambiente - CONAMA, Resolução n 20. Diário Oficial da União, 06, 1986.

COUCH, R. W.; NELSON, E. N. Effects of 2,4-D on nontarget species in Kerr Reservoir. J. Aquat. Plant. Manag., v. 20, p. 8-11, 1982.

DRAXL, R. et al. Comparison of the ecological effects of diquat on laboratory multispecies and outdoor freshwater systems. Vehr. Internat. Verein. Limnol., v. 24, p. 2269 2271, 1991.

ESTEVES, F. A. Fundamentos de limnologia. Rio de Janeiro: FINEP/Interciência, 1998. 575 p.

FAIRCHILD, F. J.; RUESSLER, D. S.; CARLSON, A. R. Comparative sensitivity of five species of macrophytes and six species of algae to atrazine, metribuzin, alachlor, and metolachlor. Environ. Toxicol. Chem., v. 17, p. 1830-1834, 1998.

FLECKNER, W. Effects of herbicides on target and nontarget organisms in aquatic systems. Verh. Internat. Verein. Limnol., v. 24, p. 2276, 1991.

GELMINI, G. A. Controle químico do aguapé (Eichhornia crassipes (Mart.) Solms) e da alface d'água (Pistia stratiotes L.). 1995. 2001 f. Dissertação (Mestrado em Fitotecnia) - Escola Superior de Agricultura "Luiz de Queiroz”, Universidade de São Paulo, Piracicaba, 1995.

GOLTERMAN, H. L.; CLYMO, R. S.; OHNSTAD, M. A. M. Methods for physical and chemical analysis of freshwaters. Blacwkweel Sci. Publ., v. 8. p. 214, 1978.

GUIMARÃES, G. L. et al. Metodologia para avaliação de impacto ambiental de macrófitas em mesocosmos. Planta Daninha, v. 21, p. 37-42, 2003.

GUITIÉRREZ, E. et al. Strategies for waterhyacinth (Eichhornia crassipes) control in Mexico. Hydrobiologia, v. 340, p. $181-185,1996$.

HOLM, L. G.; WELDON, L. W.; BLACKBURN R. D. Aquatic seeds. Science, v. 166, p. 699, 1969.

JOYCE, J. C. Chemical control. Lake Line, v. 13, p. 44-47, 1993.

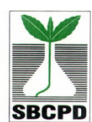

LALlANA, V. H.; SABATTINI, R. A.; LALlANA, M. D. C. Evapotranspiration from Eichhornia crassipes, Pistia stratiotes, Salvinia herzogii and Azolla caroliniana during summer in Argentina. J. Aquat. Plant Manag., v. 25, p. 48-50, 1987.

LAREO, L. Crecimiento de jacinto de água (Eichhornia crassipes (Mart) Solms Laubach), en el Trópico. Arch. Latinoam. Nutr., v. 31, p. 758, 1981.

LUGO. A. et al. Effect on the planktonic community of the chemical program used to control water hyacinth (Eichhornia crassipes) in Guadalupe Dam, Mexico. Aquatic Ecosys. Health Manag., v. 1, p. 333-343, 1998.

MARTINS, A. T. Efeitos do controle de plantas daninhas aquáticas com 2,4-D sobre alguns indicadores de qualidade da água de mesocosmos. 2001. $64 \mathrm{f}$. Monografia (Trabalho de Graduação em Agronomia) Universidade Estadual Paulista, Jaboticabal, 2001.

MARTINS, D. et al. Controle químico de Pistia stratiotes, Eichhornia crassipes e Salvinia molesta em caixas d'água. Planta Daninha, v. 20, p. 89-97, 2002. (Edição especial)

MELENDEZ, A. L. et al. Effects of diquat on freshwater microbial communities. Arch. Environ. Cont. Toxicol., v. 25 , p. $95-101,1993$.

NEVES, T.; FOLONI, L. L.; PITELLI, R. A. Controle químico do aguapé (Eichhonia crassipes). Planta Daninha, v. 20, p. 89-97, 2002. (Edição especial)

NEWMAN, J. F.; WAY, J. M. Some ecological observations on the use of paraquat and diquat as aquatic herbicides. In: BRITSH WEED CONTROL CONFERENCE, 8., 1996. Proceedings... 1996. v. 2, p. 582-585.

PENFOUND, W. T.; EARLE, T. T. Biology of water hyacinth. Ecol. Monog., v. 18, p. 449, 1948.

PETERSON, H. G. et al. Toxicity of hexazinone and diquat to green algae, diatoms, cyanobacteria and duckweed. Aquatic Toxicol., v. 39, p. 111-134, 1997.

RAJU, R. A.; REDDY, M. N. Control water hyacinth through herbicides. Ind. Farming, v. 38, p. 19-21,1988.

RITTER, A. M. et al. Characterizing aquatic ecological risks from pesticides using a diquat dibromide case study I. Probabilistic exposure estimates. Environ. Toxicol. Chem., v. 19, p. 749-759, 2000.

SCHWEGLER, B. R. Effects of sewage effluent on algal dynamics of a northern Michigan wetland. 1978. Thesis (M.S.) - The University of Michigan, Ann Arbor, 1978.

SCOTT, B. F.; DICKMANN, M. D.; HAYES, P. Effects of 2,4-D on natural phytoplankton systems in association with Myriophyllum spicatum. Vehr. Internat. Verein. Limnol., v. 22, p. 2437-2446, 1984.

Planta Daninha, Viçosa-MG, v. 23, n. 2, p. 233-242, 2005 
STEWART, K. K. Nutrient removal potentials of various aquatic plants. J. Hyacinth Control., v. 34, p. 123-29, 1970.

VELINI, E. D. Controle de plantas daninhas aquáticas. In: CONGRESSO BRASILEIRO DA CIÊNCIA DAS

PLANTAS DANINHAS, 22., 2000, Foz do Iguaçu. Palestras... Foz do Iguaçu: Sociedade Brasileira da Ciência das Plantas Daninhas, 2000. p. 137-147.

WEERT, R. V. D.; KAMERLING, G. E. Evapotranspiration of water hyacinth (Eichhornia crassipes). J. Hydrol., v. 22, p. 201-212, 1974.
WELLBORN Jr., T. L. The toxicity of nine therapeutic and herbicidal compounds to striped bass. Progr. Fish-Cult., v. 31, n. 1, p. 27-32, 1969.

WILSON, D. C.; BOND, C. E. The effects of the herbicide

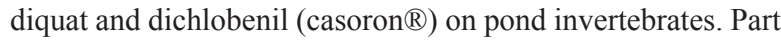
I. Acute Toxicity. Trans. Am. Fish. Soc., v. 98, p. 438-443, 1969.

WOJTALIK, T. A.; HALL, T. F.; HILL, L. O. Monitoring ecological conditions associated with wide-scale applications of DMA 2,4-D to Aquatic Environments. Pest. Monit. J., v. 4, p. 184-203, 1971. 